\title{
Far-infrared Properties of the Bright, Gravitationally Lensed Quasar J0439+1634 at $z=6.5$
}

\author{
Jinyi Yang ${ }^{1}$ (D), Bram Venemans ${ }^{2}$ (D), Feige Wang $^{3}$ (D), Xiaohui Fan ${ }^{1}$ (D), Mladen Novak ${ }^{2}$ (D), Roberto Decarli ${ }^{4}$ (D), Fabian Walter ${ }^{2}$ (iD), \\ Minghao Yue ${ }^{1}$ (D), Emmanuel Momjian ${ }^{5}$ (D), Charles R. Keeton ${ }^{6}$ (D), Ran Wang $^{7}$ (D), Ann Zabludoff ${ }^{1}$ (iD), Xue-Bing Wu ${ }^{7,8}$ (D), and \\ Fuyan Bian' 9 (iD \\ ${ }^{1}$ Steward Observatory, University of Arizona, 933 N Cherry Avenue, Tucson, AZ, USA; jinyiyang@email.arizona.edu \\ ${ }^{2}$ Max-Planck Institute for Astronomy, Königstuhl 17, D-69117 Heidelberg, Germany \\ ${ }^{3}$ Department of Physics, University of California, Santa Barbara, CA 93106-9530, USA \\ ${ }_{5}^{4}$ Osservatorio Astronomico di Bologna, via Gobetti 93/3, I-40129 Bologna, Italy \\ ${ }^{5}$ National Radio Astronomy Observatory, P. O. Box O, Socorro, NM, 87801, USA \\ ${ }^{6}$ Department of Physics and Astronomy, Rutgers University, Piscataway, NJ 08854, USA \\ ${ }^{7}$ Kavli Institute for Astronomy and Astrophysics, Peking University, Beijing 100871, People's Republic of China \\ ${ }^{8}$ Department of Astronomy, School of Physics, Peking University, Beijing 100871, People's Republic of China \\ 9 European southern Observatory, Alonso de Córdova 3107, Casilla 19001, Vitacura, Santiago 19, Chile \\ Received 2019 April 10; revised 2019 June 2; accepted 2019 June 15; published 2019 August 5
}

\begin{abstract}
We present IRAM NOrthern Extended Millimeter Array, James Clerk Maxwell Telescope/Submillimetre Common-User Bolometer Array-2 and Very Large Array (VLA) observations of the most distant known gravitationally lensed quasar, J0439+1634 at $z=6.5$. We detect strong dust emission, [C II] $158 \mu \mathrm{m}$, [C I] $369 \mu \mathrm{m}$, [O I] $146 \mu \mathrm{m}, \mathrm{CO}(6-5), \mathrm{CO}(7-6), \mathrm{CO}(9-8), \mathrm{CO}(10-9), \mathrm{H}_{2} \mathrm{O} 3_{1,2}-2_{2,1}$, and $\mathrm{H}_{2} \mathrm{O} 3_{2,1}-3_{1,2}$ lines, as well as a weak radio continuum. The strong [C II] line yields a systemic redshift of the host galaxy to be $z=6.5188 \pm 0.0002$. The magnification makes J0439+1634 the far-infrared (FIR) brightest quasar at $z>6$ known, with the brightest [C II] line yet detected at this redshift. The FIR luminosity is $(3.4 \pm 0.2) \times 10^{13} \mu^{-1} L_{\odot}$, where $\mu \sim 2.6-6.6$ is the magnification of the host galaxy, estimated based on the lensing configuration from $H S T$ imaging. We estimate the dust mass to be $(2.2 \pm 0.1) \times 10^{9} \mu^{-1} M_{\odot}$. The $\mathrm{CO}$ spectral line energy distribution using four CO lines are best fit by a two-component model of the molecular gas excitation. The estimates of molecular gas mass derived from CO lines, and atomic carbon mass are consistent, in the range of $(3.9-8.9) \times 10^{10} \mu^{-1} M_{\odot}$. The $[\mathrm{C} \mathrm{II}] /[\mathrm{C} \mathrm{I}],[\mathrm{C} \mathrm{II}] / \mathrm{CO}$, and $[\mathrm{O} \mathrm{I}] /[\mathrm{C} \mathrm{II}]$ line luminosity ratios suggest a photodissociation region model with more than one component. The ratio of $\mathrm{H}_{2} \mathrm{O} 3_{2,1}-3_{1,2}$ line luminosity to $L_{\mathrm{TIR}}$ is consistent with values in local and high-redshift ultra-/hyper-luminous infrared galaxies. The VLA observations reveal an unresolved radio continuum source, and indicate that $\mathrm{J} 0439+1634$ is a radio-quiet quasar with $R=0.05-0.17$.
\end{abstract}

Key words: cosmology: observations - galaxies: active - galaxies: high-redshift - galaxies: ISM

\section{Introduction}

As the most luminous nontransient objects, quasars at $z>6$ are unique probes for the investigation of supermassive black holes (SMBHs) and their host galaxies at early cosmic time. To date, more than 150 quasars have been discovered at $z>6$, with the highest redshift at $z=7.54$ (Bañados et al. 2018). Detections of such objects indicate the existence of up to (ten) billion $M_{\odot}$ SMBHs, and place the strongest constraints on SMBH-galaxy coevolution at early epochs. A large fraction of $z>6$ quasars have been detected with significant [C II], CO and dust emission in the rest-frame far-infrared (FIR). The submillimeter/millimeter (submm/mm) observations suggest that the vast majority of the quasar host galaxies are intensely forming stars, at rates of a few 100-1000 $M_{\odot} \mathrm{yr}^{-1}$ (e.g., Bertoldi et al. 2003a; Maiolino et al. 2005; Walter et al. 2009; Wang et al. 2013; Willott et al. 2015; Venemans et al. 2016, 2017a, 2017b, 2018; Decarli et al. 2018), supported by large reservoirs of molecular gas, resulting in bright $\mathrm{CO}$ detections (e.g., Bertoldi et al. 2003b; Walter et al. 2003, 2004; Wang et al. 2011; Venemans et al. 2017c).

Strong gravitational lensing provides a unique probe of the properties of quasars and their host galaxies. Lensing not only magnifies fluxes, but also stretches images, resulting in higher effective spatial resolution and better separation of the central quasar and host galaxy (Carilli et al. 2003; Peng et al. 2006; Treu 2010). Lensed quasars at $z \sim 3$ and 4 have been important tools for our understanding of interstellar medium (ISM) in high-redshift quasar hosts (e.g., Weiß et al. 2007). At higher redshift $(z>4.5)$, lensed quasars could be powerful tools to study those distant SMBHs and their host galaxies that are not easily resolved by current facilities. However, at $z>4.5$, only two lensed quasars at $z \sim 4.8$ (McGreer et al. 2010; More et al. 2016) were known until recently, although more than 200 quasars at $z=4-6.4$ have been observed with the Hubble Space Telescope (HST) at 0." 1 resolution (Richards et al. 2004, 2006). The lack of high-redshift lensed quasars has been attributed to either a reduced magnification bias due to a flat quasar luminosity function or a strong selection effect against lensed objects in morphology or color selection used in highredshift quasar surveys.

Our new wide-area $z \sim 7$ quasar survey (Wang et al. 2018; Yang et al. 2019) in a $20,000 \mathrm{deg}^{2}$ field has discovered the most distant known lensed quasar at $z=6.51$, UHS J043947.08+163415.7 (hereafter J0439+1634, Fan et al. 2019, hereafter Paper I). The Mg II-based redshift is $z=6.511 \pm 0.003$. In Paper I, based on our HST FR782N and FR853N imaging which has a spatial resolution of $\sim 0$ ". 075 , we fit a singular isothermal ellipsoid lensing model and obtain the best-fit, three-images lensing model of quasar. 
The high magnification $\left(\mu_{\text {quasar }}=51.3 \pm 1.4\right.$ at optical wavelengths ${ }^{10}$ ) makes this quasar the brightest observed quasar at $z>6$ at optical and near-infrared wavelengths. Follow-up observations with the James Clerk Maxwell Telescope (JCMT) indicate that $\mathrm{J} 0439+1634$ is also the brightest FIR emitter at $z>6$ (Paper I). In this paper, we report new FIR and radio observations of J0439+1634 with the IRAM NOrthern Extended Millimeter Array (IRAM/NOEMA), JCMT, and the National Science Foundation's Karl G. Jansky Very Large Array (VLA) and the detections of gas, dust emission, and radio continuum emission. We describe the observations in Section 2. The high-quality detections of dust continuum and various molecular and atomic emission lines allow detailed studies of the ISM in the quasar host, which are presented in Section 3. The summary is given in Section 4. All results below refer to a $\Lambda \mathrm{CDM}$ cosmology with parameters $\Omega_{\Lambda}=0.7$, $\Omega_{m}=0.3$, and $H_{0}=70 \mathrm{~km} \mathrm{~s}^{-1} \mathrm{Mpc}^{-1}$.

\section{Observations}

\subsection{NOEMA Observations}

We used IRAM/NOEMA to detect the [C II], CO(10-9), $\mathrm{CO}(9-8), \mathrm{CO}(7-6), \mathrm{CO}(6-5)$, [C I] $369 \mu \mathrm{m}$ (hereafter [C I]), [O I] $146 \mu \mathrm{m}$ (hereafter [O I]), and water emission lines from quasar host through two programs in the summer and winter of 2018. The observations were carried out from 2018 July to December with the array in D and C configurations, using 7-10 antennas. We used the PloyFiX correlator, which has two $7.744 \mathrm{GHz}$ sidebands separated by $7.744 \mathrm{GHz}$ (centers are separated by $15.488 \mathrm{GHz}$ ). The NOEMA data have been reduced using the latest version of the GILDAS ${ }^{11}$ software.

For the first program in the summer of 2018, we designed three setups. The $\mathrm{CO}(6-5), \mathrm{CO}(7-6)$, and [C I] lines were covered by a single setting with receiver $1(3 \mathrm{~mm})$ tuned at 107.410 GHz. The $\mathrm{CO}(9-8), \mathrm{CO}(10-9), \mathrm{H}_{2} \mathrm{O} 3_{1,2}-2_{2,1}$, and $\mathrm{H}_{2} \mathrm{O} \quad 3_{2,1}-3_{1,2}$ lines were observed with receiver $2(2 \mathrm{~mm})$ tuned at $153.395 \mathrm{GHz}$. The [C II] line was observed in receiver $3(1 \mathrm{~mm})$ with tuning frequency at $253.068 \mathrm{GHz}$. Quasars 0507 $+179, \mathrm{~J} 0440+146$ and $0446+112$ were used as amplitude and phase calibrators. Radio sources 3C84 and 3C454.3 were observed as bandpass calibrators. Stars MWC349 and LKHA101 were used to set the absolute flux density scale. The total on-source integration time in the receivers 1,2 , and 3 were $1.6,2.8$, and $1.3 \mathrm{hr}$ ( 8 antenna equivalent), respectively. The synthesized beams are 4 !" $9 \times 1$.! 9,3 !" $1 \times 2$ !" 4 , and $6 ! 2 \times 3 ! .2$ in the 1,2 , and $3 \mathrm{~mm}$ bands, respectively. The final 1,2 , and $3 \mathrm{~mm}$ cubes reach the sensitivity of 2.2, 0.7 , and $0.5 \mathrm{mJy} \mathrm{beam}^{-1}$ per $50 \mathrm{~km} \mathrm{~s}^{-1}$ channel $(1 \sigma)$. In the second program in the winter of 2018, the [C II] and [O I] lines were covered by one setting with the receiver $3(1 \mathrm{~mm})$ with a tuning frequency of $270.244 \mathrm{GHz}$. The on-source integration time in this observation is $3.13 \mathrm{hr}$ (9 antenna equivalent). The synthesized beam is 1 !" $2 \times 0$ "! 7 and final data cube reaches a $1.5 \mathrm{mJy}$ beam $^{-1}$ per $50 \mathrm{~km} \mathrm{~s}^{-1}$ sensitivity $(1 \sigma)$.

From the NOEMA observations, we obtained high-quality detections of the $\mathrm{CO}(6-5), \mathrm{CO}(7-6), \mathrm{CO}(9-8), \mathrm{CO}(10-9)$, [C II], [C I], [O I], $\mathrm{H}_{2} \mathrm{O} 3_{1,2}-2_{2,1}$, and $\mathrm{H}_{2} \mathrm{O} 3_{2,1}-3_{1,2}$ emission lines (see Figures 1 and 2). The dust continuum is significantly

\footnotetext{
${ }^{10}$ This magnification factor is derived from $H S T$ imaging for the central quasar; the total magnification for the extended host galaxy is usually significantly smaller; see Section 3.

11 http://www.iram.fr/IRAMFR/GILDAS
}

detected in all cubes. All the NOEMA detections are spatially unresolved. Measured line fluxes and continuum flux densities are summarized in Table 1 and Section 3. We only include statistical errors and the systematic flux density calibration uncertainties (of the order of $\sim 15 \%$ ) are not taken into account.

\subsection{JCMT Observations}

We observed the $450 \mu \mathrm{m}$ (observed frame, $666 \mathrm{GHz}$ ) and $850 \mu \mathrm{m}(353 \mathrm{GHz})$ dust continuum from $\mathrm{J} 0439+1634$ using the Submillimetre Common-User Bolometer Array-2 (SCUBA2; Holland et al. 2013) on the JCMT in 2018 February. The observations were carried out in Band 2 weather conditions (i.e., $0.05<\tau_{225 \mathrm{GHz}}<0.008$ ), and in "CV DAISY" mode. The effective beam size of SCUBA-2 has a diameter of 9". 8 at $450 \mu \mathrm{m}$ and 14 ." 6 at $850 \mu \mathrm{m}$. Our target was observed for $1.1 \mathrm{hr}$ on-source time (two 34 minutes scans). The data were reduced using the STARLINK SCUBA-2 pipeline for faint point sources (Chapin et al. 2013). The $1 \sigma$ sensitivities in $450 \mu \mathrm{m}$ and $850 \mu \mathrm{m}$ are $29.12 \mathrm{mJy}^{-1}$ beam $^{-1}$ and and $1.69 \mathrm{mJy} \mathrm{beam}^{-1}$, respectively. From the JCMT observations, we obtained an $850 \mu \mathrm{m}$ flux density of $26.20 \pm 1.68 \mathrm{mJy}$ and a $2 \sigma$ signal of $65.71 \pm 29.12 \mathrm{mJy}$ at $450 \mu \mathrm{m}$, which is measured at the peak flux pixel of the $850 \mu \mathrm{m}$ detection.

\subsection{VLA Observations}

We observed the radio continuum emission from J0439 +1634 using VLA DDT time in $S$ band (2-4 GHz) with the A configuration. The observed reference frequency of $3 \mathrm{GHz}$ corresponds to the rest-frame frequency of $22.6 \mathrm{GHz}$ for this quasar. The 8-bit samplers were utilized to cover the $2 \mathrm{GHz}$ span of the $S$-band receiver. The total telescope time was $3 \mathrm{hr}$ ( $2.1 \mathrm{hr}$ on-source). We used $\mathrm{J} 0440+1437$ for complex gain calibration and 3C138 for bandpass and flux density scale calibration. The data were reduced by the the Common Astronomy Software Applications package (CASA) and the VLA calibration pipeline. The synthesized beam was 0 . $79 \times 0$." 69 , and the $1 \sigma \mathrm{rms}$ noise of continuum image was $4.5 \mu \mathrm{Jy}_{\text {beam }^{-1}}$. The VLA $3 \mathrm{GHz}$ image shows an unresolved radio continuum source with a flux density of $28.9 \pm 4.5 \mu \mathrm{Jy}$.

\section{The Host Galaxy of J0439+1634}

The NOEMA spectra are shown in Figure 2 and all detections are summarized in Table 1. The detections of multi-emission lines and dust continuum at multiple frequencies allow us to investigate the properties of gas and dust in the host galaxy of $\mathrm{J} 0439+1634$. All measurements of emission lines and derived properties such as gas mass, dust mass, and star formation rate (SFR) are also listed in Table 1. The position of the quasar host derived from the [C II] emission, R.A. $=04^{\mathrm{h}} 39^{\mathrm{m}} 47^{\mathrm{s}} .10$ and decl. $=+16^{\circ} 34^{\prime} 15^{\prime \prime} \cdot 77$, is consistent with the optical location of the quasar (Paper I).

$\mathrm{J} 0439+1634$ is a compact lensed quasar with $\sim 0$." 2 separation, and has only been resolved in the HST image with a spatial resolution of 0."075 (Paper I). The HST image was designed to image quasar's Ly $\beta$ emission line. The two-orbit narrow-band imaging is relatively shallow and is dominated by emission from the central active galactic nucleus (AGN). Therefore, we can only model the lensing configuration of quasar base on HST image (see details in Paper I). The lensing configuration of host galaxy, in particular, the total magnification could be significantly different since the quasar is a point 


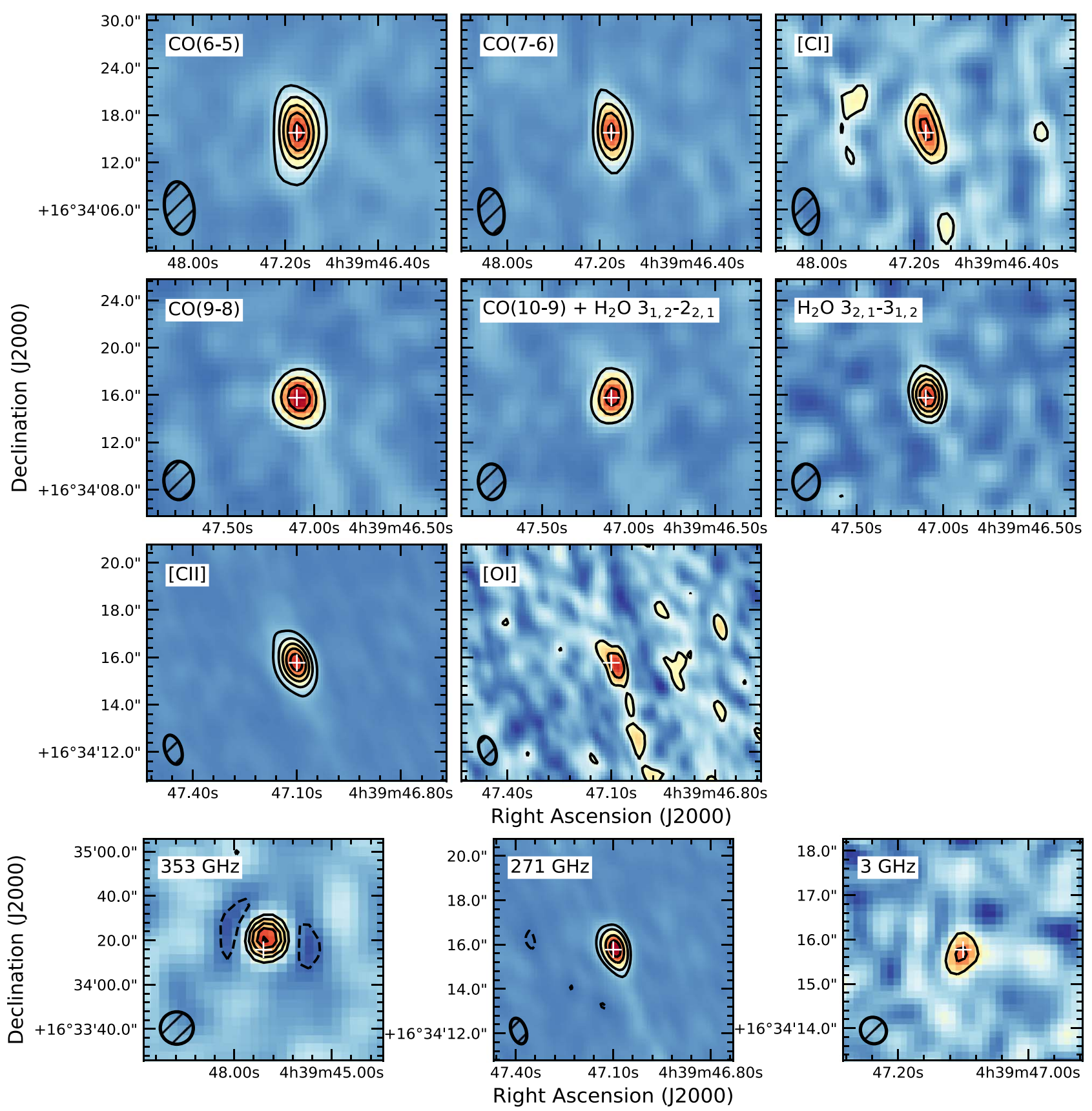

Figure 1. Maps of lines, FIR continuum, and radio continuum emissions. The line intensity map is obtained by integrating the continuum-subtracted data cubs over the velocity of each line. The white cross in each map denotes the position fitted from [C II], which is consistent with the optical coordinates. The black contours in $\mathrm{CO}(6-5), \mathrm{CO}(7-6), \mathrm{CO}(9-8)$, and $\mathrm{CO}(10-9)$ maps are $[3,6,9,12,15,18] \times \sigma$. The contours in [C I], [O I], and $\mathrm{H}_{2} \mathrm{O} 3_{2,1}-3_{1,2}$ maps are [2, 3, 4, 5, 6] $\times \sigma$. The counters in the map of $[\mathrm{C} \mathrm{II}]$ are $[5,10,15,20,25] \times \sigma$. The values of $\sigma$ in these line maps are, from upper-left $(\mathrm{CO}(6-5))$ to bottom-right $([\mathrm{O} \mathrm{I}]), 0.1,0.1,0.1,0.2$, $0.2,0.2,0.4$, and $0.3 \mathrm{Jy} \mathrm{km} \mathrm{s}^{-1}$. In the maps of continuum emission, the contours are $[3,6,9,12,15] \times \sigma$ for $S_{353 \mathrm{GHz}}(\sigma=1.7 \mathrm{mJy})$ and $S_{3} \mathrm{GHz}(\sigma=0.004 \mathrm{mJy})$ and $[10,20,40,60,80] \times \sigma$ for $S_{271 \mathrm{GHz}}(\sigma=0.2 \mathrm{mJy})$. The black dashed lines are $-3 \sigma$. Here, we only plot the $S_{271 \mathrm{GHz}}$ as a representative of dust continuum emissions detected from NOEMA observations because all detections are spatially unresolved, and the $S_{271 \mathrm{GHz}}$ map has the smallest beam size. The peak of $S_{353} \mathrm{GHz}$ emission locates $\sim 3^{\prime \prime}$ away from the optical coordinates. Considering that the beam size of $S_{353 \mathrm{GHz}}$ map is 14 ". 6 and there is no nearby source detected in all other FIR continuum maps, we believe that the $S_{353 \mathrm{GHz}}$ emission is all from J0439+1634.

source, while the host galaxy is an extended source with unclear morphology, inclination angle, and position angle. The source was not spatially resolved in our NOEMA, JCMT or VLA observations. Therefore, all measurements of intrinsic properties of the host galaxy are made based on the simulated lensing model of host galaxy and the simulation is based on the lensing configuration of quasar.

We use the isothermal ellipsoid lens model from Fan et al. (2019), including uncertainties sampled with Markov chain
Monte Carlo methods. We assume the host galaxy to be an exponential disk centered on the inferred position of the quasar. We set a grid of effective radius (0!" $1-0$ !' 25$)$ based on the typical size of quasar host galaxies at this redshift (Wang et al. 2013) and also assume a range of the projected ellipticity $(0.0-0.8)$ with random position angles. The median magnification of the host galaxy ranges from 2.6 to 4.9 across the grid of effective radius and ellipticity; over the whole grid, the $95 \%$ confidence interval for the magnification is 2.6-6.6. Note that 

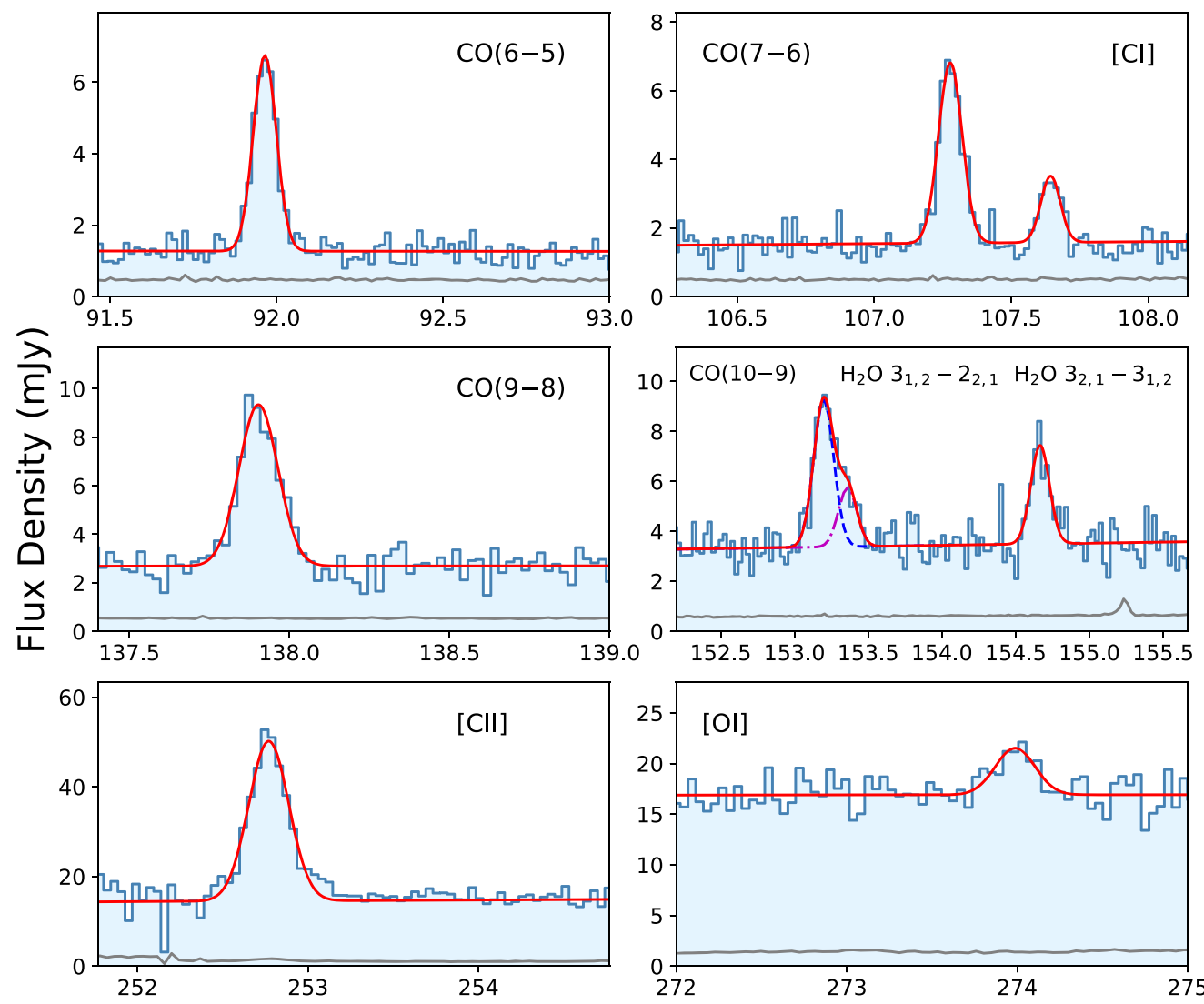

\section{Observed Frequency $(\mathrm{GHz})$}

Figure 2. NOEMA spectra of the $\mathrm{CO}(6-5), \mathrm{CO}(7-6), \mathrm{CO}(9-8), \mathrm{CO}(10-9)$, [C I], [C II], [O I], $\mathrm{H}_{2} \mathrm{O} 3_{1,2}-2_{2,1}, \mathrm{H}_{2} \mathrm{O} 3_{2,1}$ and $-3_{1,2}$ emission lines, and the underlying continuum of J0439+1634, extracted from the peak pixel in each data cube. The bin size is $\sim 50 \mathrm{~km} \mathrm{~s}^{-1}$. The gray lines show the uncertainties of the spectra. The [C II] line is the combination of two NOEMA observations. The red solid lines are the fits to spectra including a Gaussian profile for the emission lines plus continuum. The $\mathrm{CO}(10-9)$ line is blended with $\mathrm{H}_{2} \mathrm{O} 3(1,2)-2(2,1)$. A two-Gaussian fit is used for the blended line. All measured values are reported in Table 1. These are the brightest lines observed from a $z>6$ quasar host.

the total magnification of the host galaxy is significantly smaller than that of the central quasar $\left(\mu_{\text {quasar }} \sim 51\right)$ because the host galaxy is extended. For the rest of the paper, we will use $\mu$ as the magnification factor of the host galaxy. Our forthcoming high resolution ALMA imaging will directly resolve the host galaxy into a lensed arc, and allow detailed lensing modeling to measure the size and shape of the quasar host galaxy, and to derive its magnification factor accurately.

In a lensed system, the different angular size of extended emissions from different lines or continuum (e.g., if the dust emission is more compact than the [C II] emitting region) could result in differential lensing effect. Differential lensing could also distort the velocity profile of emission lines, depending on how the kinematic structure of the source couples with the lensing magnification pattern (Rivera et al. 2019; Yang et al. 2019). The emission lines of J0439 from NOEMA observations are well fitted by a single Gaussian profile, except for the blended $\mathrm{CO}(10-9)$ and $\mathrm{H}_{2} \mathrm{O}_{3} 3_{1,2}-2_{2,1}$ and the possible wings of [C II] line (see Figure 2 and Section 3.1). Therefore, we do not include any discussion of this effect in this paper, and until we resolve the host we assume the same magnification of all FIR emission. The intrinsic properties measured in this paper, especially the ratios between different parameters, could nevertheless be affected by differential lensing. Giving these complications, in this paper, we simply report the observed values of quasar host galaxy properties with a common magnification factor $\mu$.

\subsection{Continuum Emission and Infrared (IR) Luminosity}

We detect dust continuum at observed frame frequencies of $352.7 \mathrm{GHz}$ from JCMT, and 271, 254, 239, 155, 139, 109, and $93 \mathrm{GHz}$ from NOEMA. The dust continuum from the NOEMA observations used in this section are measured by averaging spectra over all channels within the line free regions in each sideband. The dust continuum is detected with high signal-toratio in the NOEMA spectra, as shown in Figures 1 and 2. All flux densities are listed in Table 1. The JCMT observation at $666.2 \mathrm{GHz}$ show a $3 \sigma$ upper limit.

To estimate the FIR rest-frame 42.5-122.5 $\mu$ m luminosity, $L_{\mathrm{FIR}}$, total infrared (TIR, rest-frame $8-1000 \mu \mathrm{m}$ ) luminosity $L_{\mathrm{TIR}}$ and the dust mass, we fit the dust emission with an optically thin graybody following the literature (e.g., Beelen et al. 2006). We fit a set of models with different dust temperatures $T_{d}$ and fixed the emissivity index $\beta=1.6$ or 1.95 , as shown in Figure 3. We take the effect of the cosmic microwave background (CMB) on the dust emission into account (e.g., da Cunha et al. 2013). We exclude the $666.2 \mathrm{GHz}$ upper limit for the model fitting. We obtain a best-fit (least $\chi^{2}$ ) dust temperature of $T_{d}=39 \pm 9 \mathrm{~K}$ at fixed $\beta=1.6$, consistent with the canonical value of $T_{d}=47 \mathrm{~K}$ (Beelen et al. 2006). If 
Table 1

Observed and Derived Properties of J0439+1634

\begin{tabular}{|c|c|}
\hline R.A. (J2000) & $04^{\mathrm{h}} 39^{\mathrm{m}} 47^{\mathrm{s}} .10$ \\
\hline Decl. (J2000) & $+16^{\circ} 34^{\prime} 15^{\prime \prime} \cdot 77$ \\
\hline$\left.z_{[[\mathrm{C}} \mathrm{II}\right]$ & $6.5188 \pm 0.0002$ \\
\hline$F_{[\mathrm{C} \mathrm{II}]}\left(\mathrm{Jy} \mathrm{km} \mathrm{s}^{-1}\right)$ & $11.7 \pm 0.8$ \\
\hline $\mathrm{FWHM}_{[\mathrm{C} \mathrm{II}]}\left(\mathrm{km} \mathrm{s}^{-1}\right)$ & $328.1 \pm 18.0$ \\
\hline$F_{\mathrm{CO}(6-5)}\left(\mathrm{Jy} \mathrm{km} \mathrm{s}^{-1}\right)$ & $1.5 \pm 0.1$ \\
\hline $\mathrm{FWHM}_{\mathrm{CO}(6-5)}\left(\mathrm{km} \mathrm{s}^{-1}\right)$ & $266.0 \pm 15.8$ \\
\hline$F_{\mathrm{CO}(7-6)}\left(\mathrm{Jy} \mathrm{km} \mathrm{s}^{-1}\right)$ & $1.5 \pm 0.1$ \\
\hline $\mathrm{FWHM}_{\mathrm{CO}(7-6)}\left(\mathrm{km} \mathrm{s}^{-1}\right)$ & $282.7 \pm 18.0$ \\
\hline$F_{\mathrm{CO}(9-8)}\left(\mathrm{Jy} \mathrm{km} \mathrm{s}^{-1}\right)$ & $2.1 \pm 0.2$ \\
\hline $\mathrm{FWHM}_{\mathrm{CO}(9-8)}\left(\mathrm{km} \mathrm{s}^{-1}\right)$ & $312.5 \pm 22.4$ \\
\hline$F_{\mathrm{CO}(10-9)}\left(\mathrm{Jy} \mathrm{km} \mathrm{s}^{-1}\right)$ & $1.9 \pm 0.2$ \\
\hline $\mathrm{FWHM}_{\mathrm{CO}(10-9)}{ }^{\mathrm{a}}\left(\mathrm{km} \mathrm{s}^{-1}\right)$ & $312.5 \pm 17.9$ \\
\hline$F_{[\mathrm{C} \mathrm{I}]}\left(\mathrm{Jy} \mathrm{km} \mathrm{s}^{-1}\right)$ & $0.5 \pm 0.1$ \\
\hline $\mathrm{FWHM}_{[\mathrm{C} \mathrm{I}]}\left(\mathrm{km} \mathrm{s}^{-1}\right)$ & $232.0 \pm 36.6$ \\
\hline$F_{\left[\mathrm{O}_{\mathrm{I}}\right]}\left(\mathrm{Jy} \mathrm{km} \mathrm{s}^{-1}\right)$ & $1.4 \pm 0.3$ \\
\hline $\mathrm{FWHM}_{[\mathrm{O} \mathrm{I}]}\left(\mathrm{km} \mathrm{s}^{-1}\right)$ & $298.9 \pm 56.1$ \\
\hline$F_{\mathrm{H}_{2} \mathrm{O}(312-221)}\left(\mathrm{Jy} \mathrm{km} \mathrm{s}^{-1}\right)$ & $0.9 \pm 0.2$ \\
\hline $\mathrm{FWHM}_{\mathrm{H}_{2} \mathrm{O}(312-221)}{ }^{\mathrm{b}}\left(\mathrm{km} \mathrm{s}^{-1}\right)$ & $288.4 \pm 42.5$ \\
\hline$F_{\mathrm{H}_{2} \mathrm{O}(321-312)}\left(\mathrm{Jy} \mathrm{km} \mathrm{s}^{-1}\right)$ & $1.1 \pm 0.2$ \\
\hline $\mathrm{FWHM}_{\mathrm{H}_{2} \mathrm{O}(321-312)}\left(\mathrm{km} \mathrm{s}^{-1}\right)$ & $288.4 \pm 44.4$ \\
\hline$S_{666 \mathrm{GHz}}(\mathrm{mJy})$ & $<87.3$ \\
\hline$S_{353 \mathrm{GHz}}(\mathrm{mJy})$ & $26.2 \pm 1.7$ \\
\hline$S_{271 \mathrm{GHz}}(\mathrm{mJy})$ & $16.9 \pm 0.1$ \\
\hline$S_{255 \mathrm{GHz}}(\mathrm{mJy})$ & $15.5 \pm 0.1$ \\
\hline$S_{239 \mathrm{GHz}}(\mathrm{mJy})$ & $14.0 \pm 0.1$ \\
\hline$S_{155 \mathrm{GHz}}(\mathrm{mJy})$ & $3.5 \pm 0.04$ \\
\hline$S_{139 \mathrm{GHz}}(\mathrm{mJy})$ & $2.7 \pm 0.03$ \\
\hline$S_{109 \mathrm{GHz}}(\mathrm{mJy})$ & $1.6 \pm 0.03$ \\
\hline$S_{93 \mathrm{GHz}}(\mathrm{mJy})$ & $1.3 \pm 0.03$ \\
\hline$S_{3 \mathrm{GHz}}(\mu \mathrm{Jy})$ & $28.9 \pm 4.5$ \\
\hline$\mu^{\mathrm{c}}$ & $2.6-6.6$ \\
\hline$L_{\mathrm{FIR}}\left(L_{\odot}\right)$ & $(3.4 \pm 0.2) \times 10^{13} \mu^{-1}$ \\
\hline$L_{\mathrm{TIR}}\left(L_{\odot}\right)$ & $(4.8 \pm 0.2) \times 10^{13} \mu^{-1}$ \\
\hline$L_{[\mathrm{C} \mathrm{II]}}\left(L_{\odot}\right)$ & $(1.2 \pm 0.1) \times 10^{10} \mu^{-1}$ \\
\hline$L_{[\mathrm{C} \text { II] }}^{\prime}\left(\mathrm{K} \mathrm{km} \mathrm{s}^{-1} \mathrm{pc}^{2}\right)$ & $(5.7 \pm 0.4) \times 10^{10} \mu^{-1}$ \\
\hline$L_{[\mathrm{C} \mathrm{I}]}\left(L_{\odot}\right)$ & $(2.0 \pm 0.4) \times 10^{8} \mu^{-1}$ \\
\hline$L_{[\mathrm{C} \mathrm{I}]}^{\prime}\left(\mathrm{K} \mathrm{km} \mathrm{s}^{-1} \mathrm{pc}^{2}\right)$ & $(1.2 \pm 0.2) \times 10^{10} \mu^{-1}$ \\
\hline $\operatorname{SFR}_{\mathrm{TIR}}\left(M_{\odot} \mathrm{yr}^{-1}\right)$ & $7080 \mu^{-1}$ \\
\hline $\operatorname{SFR}_{[\mathrm{C} \text { II }]}\left(M_{\odot} \mathrm{yr}^{-1}\right)$ & $1000-6000 \mu^{-1.18}$ \\
\hline$M_{d}\left(M_{\odot}\right)$ & $(2.2 \pm 0.1) \times 10^{9} \mu^{-1}$ \\
\hline$M_{\mathrm{C} \text { I }}\left(M_{\odot}\right)$ & $(2.6 \pm 0.5) \times 10^{7} \mu^{-1}$ \\
\hline$M_{\mathrm{C}^{+}}\left(M_{\odot}\right)$ & $(3.7 \pm 0.3) \times 10^{7} \mu^{-1}$ \\
\hline$M_{\mathrm{H}_{2}, \mathrm{CO}}\left(M_{\odot}\right)^{\mathrm{d}}$ & $5.4 \times 10^{10} \mu^{-1}$ \\
\hline$M_{\mathrm{H}_{2}, \mathrm{C} \text { I }}\left(M_{\odot}\right)^{\mathrm{e}}$ & $(3.9-8.9) \times 10^{10} \mu^{-1}$ \\
\hline
\end{tabular}

Notes.

a We fix the FWHM of $\mathrm{CO}(10-9)$ line to that measured from $\mathrm{CO}(9-8)$ line.

${ }^{\mathrm{b}}$ We fix the FWHM of $\mathrm{H}_{2} \mathrm{O} 3_{1,2}-2_{2,1}$ line to that measured from $\mathrm{H}_{2} \mathrm{O} 3_{2,1}-3_{1,2}$ line.

c $\mu$ is the lens magnification of host galaxy (see Section 3).

d Molecular gas mass derived from the $\mathrm{CO}(1-0)$ luminosity, assuming a luminosity-to-gas mass conversion factor of $\alpha=0.8 M_{\odot}\left(\mathrm{K} \mathrm{km} \mathrm{s}^{-1} \mathrm{pc}^{2}\right)^{-1}$.

e Molecular gas mass derived from the atomic carbon mass, assuming a carbon abundance of $\mathrm{X}[\mathrm{CI}]=(8.4 \pm 3.5) \times 10^{-5}$.

we fix the $T_{d}$ to $47 \mathrm{~K}$, the best-fitting value of $\beta$ is $1.4 \pm 0.2$. The temperature strongly depends on the flux densities measured near the peak of the dust spectral energy distribution (SED). Stronger constraints on the dust temperature require additional photometry at higher frequency. Therefore, we assume the $T_{d}=47 \mathrm{~K}$ and $\beta=1.6$ for related measurements.

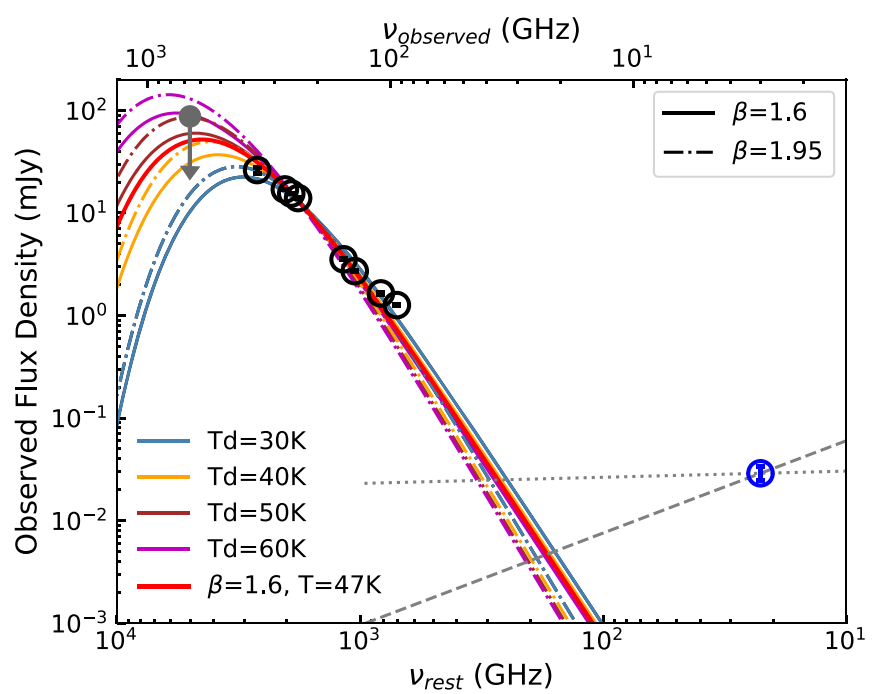

Figure 3. FIR and radio spectral energy distribution of J0439+1634. Black circles represent measurements from the JCMT and NOEMA observations of the dust continuum. The gray point with arrow denote the $3 \sigma$ upper limit at $666 \mathrm{GHz}$. The blue circle is the $3 \mathrm{GHz}$ detection from the VLA, which shows the radio emission. Two gray lines are the radio continua with different slopes, $\alpha=-0.06$ (dotted) and $\alpha=-0.9$ (dashed), assuming the slope range based on previous results of quasar $\mathrm{J} 0100+2802$ (Wang et al. 2016). The red line is the typical dust emission model with $T_{d}=47 \mathrm{~K}$ and $\beta=1.6$ (Beelen et al. 2006). The dotted-dashed lines are models with $\beta=1.95$. A series of models with different $T_{d}$ are plotted for comparison. With fixed $\beta=1.6$, the best-fitting $T_{d}$ is $39 \pm 9 \mathrm{~K}$, which changes to $T_{d}=29 \pm 6 \mathrm{~K}$ with $\beta=1.95$.

With this assumption, the luminosities derived from the graybody model are $L_{\mathrm{FIR}}=(3.4 \pm 0.2) \times 10^{13} \mu^{-1} L_{\odot}$ and $L_{\mathrm{TIR}}=(4.8 \pm 0.2) \times 10^{13} \mu^{-1} L_{\odot}$. The derived FIR luminosity is the highest for all known high-redshift quasars (observed value before correcting for lensing magnification).

We estimate the SFR from the $L_{\mathrm{TIR}}$ based on the local scaling relation from Murphy et al. (2011) and assuming the $L_{\text {TIR }}$ is dominated by star formation. We obtain an SFR of $7080 \mu^{-1} M_{\odot} \mathrm{yr}^{-1}$. Any contribution from quasar to the IR luminosity will result in an overestimation of the SFR. The dust mass based on the assumption of dust emission model is $M_{d}=2.2 \pm 0.1 \times 10^{9} \mu^{-1} M_{\odot}$, where the uncertainty is only from fitting. Some recent works suggest that the relatively lowmetallicity galaxy template Haro 11 could provide a better fit to the IR SED of high-redshift quasar (Lyu et al. 2016; De Rossi et al. 2018). As a comparison, we also fit the observed data with the Haro 11 template and a AGN template from Lyu et al. (2016), which results in a 1.6 times higher TIR luminosity, consistent with the systematic difference between the optically thin graybody model and Haro 11 template (De Rossi et al. 2018).

The VLA $3 \mathrm{GHz}$ detection represents the rest-frame $22.6 \mathrm{GHz}$ radio emission from quasar. We calculate the radio loudness by adopting the definition of $R_{2500}=f_{\nu}, 5 \mathrm{GHz} / f_{\nu, 2500} \AA$, where the $f_{\nu, 2500 \AA}$ is the optical flux density at rest frame $2500 \AA$, measured from the NIR spectrum of J0439+1634. The rest frame $5 \mathrm{GHz}$ flux density $f_{\nu, 5 \mathrm{GHz}}$ is estimated from the VLA detection assuming a power-law radio continuum $\left(f_{\nu} \propto \nu^{\alpha}\right)$. The radio loudness is constrained to be $R=0.05$ with a flat continuum $\alpha=-0.06$, and $R=0.17$ with a steep slope of $\alpha=-0.9$, which indicates that $\mathrm{J} 0439+1634$ is a radio-quiet quasar. Based on the current lensing models, if the radio emission is dominated by the quasar, the intrinsic radio emission $L_{\nu, 1.4 \mathrm{GHz}}$ will be $10^{-3.9}-10^{-2.9} L_{\odot} \mathrm{Hz}^{-1}$ assuming a slope of 
$\alpha=-0.06$ to -0.9 . On the other hand, based on the radio-FIR relation (Yun et al. 2001; Wang et al. 2007), the radio emission from host could be expected to be $L_{\nu, 1.4 \mathrm{GHz}} \gtrsim 10^{-3} L_{\odot} \mathrm{Hz}^{-1}$ when assuming the $L_{\mathrm{FIR}}$ is dominated by star formation and considering the scatter of the radio-FIR relation. This result suggests that the host galaxy contributes a significant fraction of radio emission, which could be higher than or comparable to that from quasar. Currently, only one frequency detection of radio continuum and the uncertain lensing configuration limit the more detailed investigation of radio emission properties.

\section{2. [C II] Emission Line}

The [C II] $158 \mu \mathrm{m}$ emission line is detected from NOEMA observations (Figure 2) with a line peak flux density of $\sim 36 \mathrm{mJy}$, the brightest [C II] line detected from any quasar at $z \gtrsim 6$ (e.g., Decarli et al. 2018). We extract the spectrum from the peak pixel in the data cube and fit the emission line with a Gaussian profile. The [C II] line tentatively shows wings that can not be matched by a single Gaussian fit (see Figure 2). Due to the relatively low signal-to-noise ratio $(\mathrm{S} / \mathrm{N})$ at the wings, we adopt a single Gaussian profile as the best fit. Future high $\mathrm{S} / \mathrm{N}$ observations are needed to confirm and to investigate the nature of the extended wing of [C II] line. The redshift measured from [C II] line is $z_{[\mathrm{C} \mathrm{II}]}=6.5188 \pm 0.0002$, which is slightly higher than the Mg IIbased redshift $(z=6.511 \pm 0.003)$, indicating a $311 \mathrm{~km} \mathrm{~s}^{-1}$ blueshift of the $\mathrm{Mg}$ II line. This blueshift is similar to shifts between $\mathrm{Mg}$ II and [C II] found in other $z>6$ quasars (e.g., several hundred $\mathrm{km} \mathrm{s}^{-1}$; Mazzucchelli et al. 2017; Decarli et al. 2018). We obtain a line flux of $F_{[\mathrm{C} \mathrm{II}]}=11.7 \pm 0.8 \mathrm{Jy} \mathrm{km} \mathrm{s}^{-1}$, and a $\mathrm{FWHM}_{[\mathrm{CI}]}=328.1 \pm 18.0 \mathrm{~km} \mathrm{~s}^{-1}$, corresponding to a line luminosity of $L_{[\mathrm{C} \text { II] }}=(1.2 \pm 0.1) \times 10^{10} \mu^{-1} L_{\odot} \quad\left(L_{[\mathrm{C} \text { II] }}^{\prime}\right.$ $\left.=(5.7 \pm 0.4) \times 10^{10} \mu^{-1} \mathrm{~K} \mathrm{~km} \mathrm{~s}^{-1} \mathrm{pc}^{2}\right)$.

The detection of the [C II] line allows us to derive a $L_{[\mathrm{C} \text { II] }}$ -based SFR. We use the SFR- $L_{[\mathrm{C} \text { II] }}$ relations for high-redshift $(z>0.5)$ galaxies from De Looze et al. (2014):

$$
\mathrm{SFR}_{[\mathrm{C} \text { II] }} / M_{\odot} \mathrm{yr}^{-1}=3.0 \times 10^{-9}\left(L_{[\mathrm{C} \mathrm{II}]} / L_{\odot}\right)^{1.18},
$$

which has a systematic uncertainty of a factor of $\sim 2.5$. Therefore, we obtain an observed SFR $_{[\mathrm{C} \mathrm{II}]}$ of $\sim 1000-6000$ $\mu^{-1.18} M_{\odot} \mathrm{yr}^{-1}$, slightly lower than the $\mathrm{SFR}_{\mathrm{TIR}}$. The [C II] -toFIR luminosity ratio $L_{[\mathrm{C} \mathrm{II}]} / L_{\mathrm{FIR}}$ is $(3.1-4.1) \times 10^{-4}$, within the range of the $L_{[\mathrm{C} \mathrm{II}]} / L_{\mathrm{FIR}}$ distribution of previously known $z \gtrsim 6$ quasars (Venemans et al. 2017b; Decarli et al. 2018).

The [C II] line also enables us to derive the mass of singly ionized carbon $M_{C^{+}}$. Following Venemans et al. (2017c), we calculate the $M_{C^{+}}$using

$$
\begin{aligned}
M_{\mathrm{C}^{+}} / M_{\odot} & =C m_{\mathrm{C}} \frac{8 \pi k \nu_{0}^{2}}{h c^{3} A} Q\left(T_{\mathrm{ex}}\right) \frac{1}{4} e^{91.2 / T_{\mathrm{ex}}} L_{[\mathrm{C} \text { II }]}^{\prime} \\
& =2.92 \times 10^{-4} Q\left(T_{\mathrm{ex}}\right) \frac{1}{4} e^{91.2 / T_{\mathrm{ex}}} L_{[\mathrm{C} \mathrm{II}]}^{\prime},
\end{aligned}
$$

where $C$ is the conversion between $\mathrm{pc}^{2}$ and $\mathrm{cm}^{2}, m_{C}$ is the mass of a carbon atom, $A=2.29 \times 10^{-6} \mathrm{~s}^{-1}$ is the Einstein coefficient (Nussbaumer \& Storey 1981), $Q\left(T_{\mathrm{ex}}\right)=2+4 e^{-91.2 / T_{\mathrm{ex}}}$ is the C II partition function, $T_{\mathrm{ex}}$ is the excitation temperature, and $L_{\text {[C II] }}^{\prime}$ is the line luminosity in $\mathrm{K} \mathrm{km} \mathrm{s}^{-1} \mathrm{pc}^{2}$. We also assume the $T_{\text {ex }} \gtrsim 100 \mathrm{~K}$ (e.g., Meijerink et al. 2007; Venemans et al. 2017b). The derived $M_{\mathrm{C}^{+}}$is $(3.7 \pm 0.3) \times 10^{7} \mu^{-1}$ with $T_{\mathrm{ex}}=100 \mathrm{~K}$ and $3.1 \times 10^{7} \mu^{-1}$ if $T_{\mathrm{ex}}=200 \mathrm{~K}$.
Although the NOEMA observations do not resolve the source, the $[\mathrm{C} \mathrm{II}]$ line could provide a constraint on the dynamical mass to first order. In a dispersion-dominated system, the dynamical mass can be estimated as $M_{\text {dyn }}=3 R_{[\mathrm{C} \text { II] }} \sigma^{2} / 2 G$. We adopt the velocity dispersion $\sigma$ estimated from the $\mathrm{FWHM}_{[\mathrm{C} \mathrm{I}]}$ of Gaussian fit to the emission line and assume a radius of the [C II] line emitting region of $R_{[\mathrm{C} \mathrm{II}]}=2.8 \mathrm{kpc}$, the mean value of the $z \gtrsim 6$ quasar sample from Decarli et al. (2018), which results in a dynamical mass of $M_{\mathrm{dyn}} \sim 1.9 \times 10^{10} M_{\odot}$. Alternatively, the [C II] emission might originate from a thin rotating disk with an inclination angle $i$ (e.g., Wang et al. 2013; Willott et al. 2015; Venemans et al. 2016, 2017b). In this case, the dynamical mass is computed as $M_{\mathrm{dyn}}=R_{[\mathrm{C} \mathrm{II}]}\left(0.75 \mathrm{FWHM}_{[\mathrm{C} \mathrm{II}]} / \sin (i)\right)^{2} / G$ (e.g., Ho 2007; Wang et al. 2013). Since we assume an intrinsic radius $R_{[\mathrm{C} \mathrm{II}]}$ of $2.8 \mathrm{kpc}$, the host galaxy dynamical mass is estimated directly from the line width here and not affected by the value of $\mu$ (except for the potential differential lensing effect). The median value of the inclination angle of $z \sim 6$ quasars is $i=55^{\circ}$ (e.g., Wang et al. 2013; Venemans et al. 2017b). Adopting this inclination angle, we obtain $M_{\text {dyn }} \sim 5.9 \times 10^{10} M_{\odot}$. The lensing-corrected $\mathrm{Mg}$ II-based black hole mass of J0439+1634 is $6.89 \times 10^{8} M_{\odot}$ (Paper I). Thus, the $M_{\mathrm{BH}} / M_{\mathrm{dyn}}$ ratio is $\sim 0.011-0.036$, which is consistent with the measurements of $z \gtrsim 6$ quasar sample (Decarli et al. 2018) and higher than the typical value $\sim 0.002$ of local galaxies.

\subsection{CO Lines and Molecular Gas Mass}

With the NOEMA observations, we detect four $\mathrm{CO}$ emission lines: $\mathrm{CO}(6-5), \mathrm{CO}(7-6), \mathrm{CO}(9-8)$, and $\mathrm{CO}(10-9)$ (Figure 2). To measure the line luminosities, we fit each emission line with a Gaussian profile. The measured line fluxes and FWHMs are summarized in Table 1 . The $\mathrm{CO}(10-9)$ line is blended with water vapor emission $\mathrm{H}_{2} \mathrm{O} 3_{1,2}-2_{2,1}$ line. We fit the blended line with two Gaussian components and fixed FWHMs. We fix the FWHM of one component to the value measured from $\mathrm{CO}(9-8)$ line and assume the other one to be the same FHWM to that of $\mathrm{H}_{2} \mathrm{O} 3_{2,1}-3_{1,2}$ line. With multiple $\mathrm{CO}$ lines, we can investigate the physical condition (e.g., temperature and density) of molecular gas through the CO spectral line energy distribution (COSLED). We fit the COSLED with large velocity gradient (LVG) model using $\chi^{2}$ fitting. We use the one-dimensional non-LTE (Local Thermodynamic Equilibrium) radiative transfer code, RADEX, developed by van der Tak et al. (2007). We use both a single LVG model and a two-component model for the fitting, as shown in Figure 4. We set the background temperature to be the CMB temperature at $z=6.5188$ of $\sim 20.5 \mathrm{~K}$. The best fit of one-component model has temperature $T=40 \mathrm{~K}$ and gas density $n=10^{5} \mathrm{~cm}^{-3}$, while the best-fit two-component model includes a "cold" component with $T=23 \mathrm{~K}$ and $n=10^{4.1} \mathrm{~cm}^{-3}$, and a "warm" component with $T=140 \mathrm{~K}$ and $n=10^{4.5} \mathrm{~cm}^{-3}$.

The current models can be used to estimate the $\mathrm{CO}(1-0)$ line luminosity and further constrain the molecular gas mass, although the COSLED is still uncertain due to the lack of low- $J$ CO line. The one-component model results in a $\mathrm{CO}(1-0)$ line flux of $F_{\mathrm{CO}(1-0)} \sim 0.04 \mathrm{Jy} \mathrm{km} \mathrm{s}^{-1}$ and a line luminosity of $L_{\mathrm{CO}(1-0)} \sim 2.5 \times 10^{6} L_{\odot}\left(L_{\mathrm{CO}(1-0)}^{\prime} \sim 5.1 \times 10^{10} \mathrm{~K} \mathrm{~km} \mathrm{~s}^{-1} \mathrm{pc}^{2}\right)$. The two-component model provides $F_{\mathrm{CO}(1-0)} \sim 0.05 \mathrm{Jy} \mathrm{km} \mathrm{s}^{-1}$ and $L_{\mathrm{CO}(1-0)} \sim 3.3 \times 10^{6} L_{\odot}\left(L_{\mathrm{CO}(1-0)}^{\prime} \sim 6.7 \times 10^{10} \mathrm{~K} \mathrm{~km} \mathrm{~s}^{-1} \mathrm{pc}^{2}\right)$. Therefore, the $[\mathrm{C}$ II]-to- $\mathrm{CO}(1-0)$ luminosity ratios from onecomponent and two-component models are 5200 and 3900, respectively. The [C II]-to- $\mathrm{CO}(1-0)$ luminosity ratio of three $z \sim 6$ 


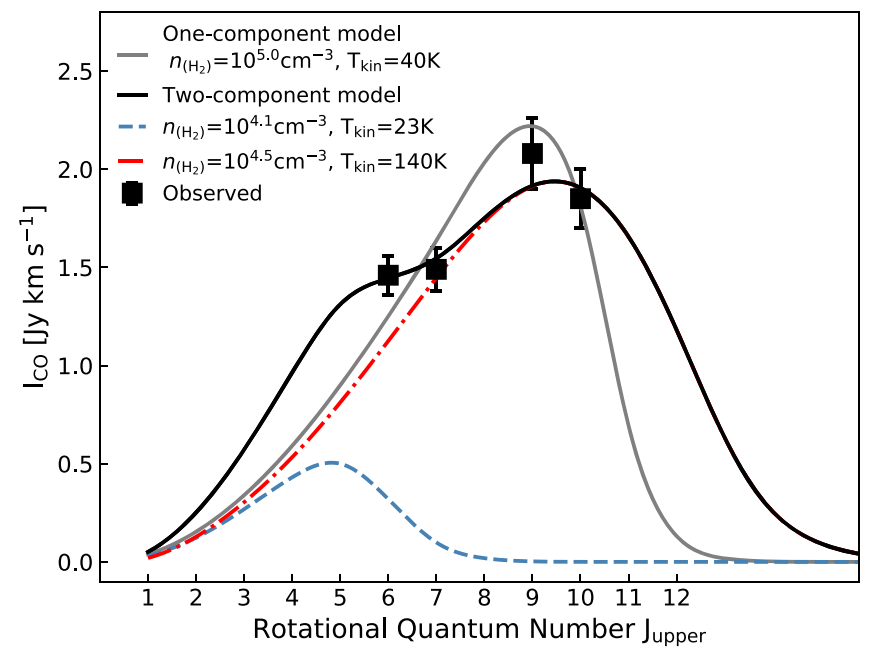

Figure 4. COSLED of J0439+1634. Four black squares with errors are observed data from NOEMA. The gray solid line is the best fit of a single LVG model, with $T=40 \mathrm{~K}$ and $n=10^{5} \mathrm{~cm}^{-3}$. The black solid line represents the two-component model: a "cold" component (blue dashed line) with $T=23 \mathrm{~K}$ and $n=10^{4.1} \mathrm{~cm}^{-3}$, and a "warm" component (red dotted-dashed line) with $T=140 \mathrm{~K}$ and $n=10^{4.5} \mathrm{~cm}^{-3}$.

quasars given by Venemans et al. (2017c) is 2500-4200, and the typical value measured from local and $z>2$ star-forming galaxies is $\sim 4100$ (Stacey et al. 1991; Gullberg et al. 2015). The [C II]-to$\mathrm{CO}(1-0)$ luminosity ratio derived from two-component model is more consistent with these previous measurements, although the one-component model can not be ruled out either. We adopt the two-component model to estimate the molecular gas mass $M_{\mathrm{H}_{2}, \mathrm{CO}}$ using $M_{\mathrm{H}_{2}, \mathrm{CO}}=\alpha L_{\mathrm{CO}(1-0)}^{\prime}$ and assuming the luminosity-to-gas mass conversion factor of $\alpha=0.8 M_{\odot}\left(\mathrm{K} \mathrm{km} \mathrm{s}^{-1} \mathrm{pc}^{2}\right)^{-1}$. The molecular gas mass is estimated as $5.4 \times 10^{10} \mu^{-1} M_{\odot}$, consistent with the value measured from atomic gas mass (see Table 1).

\section{4. [C I] and Atomic Carbon Mass}

The [CI] emission line has a line luminosity of $L_{[\mathrm{C} \mathrm{I}]}=2.04 \pm 0.41 \times 10^{8} \mu^{-1} L_{\odot} \quad\left(L_{[\mathrm{C} \text { I }]}^{\prime}=1.20 \pm 0.24 \times\right.$ $10^{10} \mu^{-1} \mathrm{~K} \mathrm{~km} \mathrm{~s}^{-1} \mathrm{pc}^{2}$ ) and $\mathrm{FWHM}_{[\mathrm{C} \mathrm{I}]}=232.04 \pm 36.59$ $\mathrm{km} \mathrm{s}^{-1}$. We can estimate the atomic carbon mass based on the $[\mathrm{CI}$ line using the following relation between [C I] brightness and the mass in neutral carbon from Weiß et al. $(2003,2005)$ assuming the [C I] emission is optically thin:

$$
M_{\mathrm{CI}} / M_{\odot}=4.566 \times 10^{-4} Q\left(T_{\mathrm{ex}}\right) \frac{1}{5} e^{T_{2} / T_{\mathrm{ex}}} L_{[\mathrm{C} \text { I }]}^{\prime},
$$

where $Q\left(T_{\mathrm{ex}}\right)=1+3 e^{-T_{1} / T_{\mathrm{ex}}}+5 e^{-T_{2} / T_{\mathrm{ex}}}$ is the C I partition function, $T_{1}=23.6 \mathrm{~K}$ and $T_{2}=62.5 \mathrm{~K}$ are the energies above the ground state. We assume the excitation temperature to $T_{\text {ex }}=30 \mathrm{~K}$ (see, e.g., Walter et al. 2011) and obtain the atomic carbon mass of $M_{\mathrm{C} \text { I }}=(2.6 \pm 0.5) \times 10^{7} \mu^{-1} M_{\odot}$. Assuming the atomic carbon abundance $\left(\mathrm{X}[\mathrm{CI}]=M_{\mathrm{C}} /\left(6 M_{\mathrm{H}_{2}}\right)\right)$ to be $\mathrm{X}$ $[\mathrm{C} \mathrm{I}]=(8.4 \pm 3.5) \times 10^{-5}$, following Walter et al. $(2011)$ and Venemans et al. (2017c), we can estimate an independent molecular gas of $M_{\mathrm{H}_{2}, \mathrm{C} \text { I }}=(3.9-8.9) \times 10^{10} \mu^{-1} M_{\odot}$. This is consistent with the molecular gas mass estimated from $\mathrm{CO}$ lines derived in the previous section.

\subsection{The Characteristics of the ISM}

The multiple FIR lines could place constraints on the physical properties of the ISM in the host galaxy through different line ratios. We compare the luminosity ratios of [C II]/[C I], [C II]/CO, and [O I]/ [C II] observed from J0439 +1634 to the predictions by the photodissociation regions (PDR) model from PDR Toolbox (Kaufman et al. 1999, 2006; Pound \& Wolfire 2008). A one-sided illuminated slab geometry is adopted in this PDR model. In reality, assuming spherical clouds, we would detect optical thin emission from both the front and the back of the cloud, but only detect optically thick emission from the front. Therefore, when we apply this model to observed data, we need to multiply the observed optically thick line flux by two or divide the observed optically thin emission by two to match the model. Here we divide the optically thin [C II] line emission by a factor of two. We also assume the fraction of [C II] emitted from PDR is $\sim 80 \%$, considering the dust temperature $T_{d}$ and the correlations of local luminous infrared galaxies from Díaz-Santos et al. (2017).

The overlapped region of line ratios will constrain the gas density $n$ and the incident far-ultraviolet radiation field $G$ (in units of the Habing Field, $G_{0}, 1.6 \times 10^{-3} \mathrm{ergs} \mathrm{cm}^{-2} \mathrm{~s}^{-1}$ ). As shown in Figure 5 (top panel), the four ratios of $L_{[\mathrm{C} \mathrm{II}]} / L_{\mathrm{CO}}$ overlap in a region with high radiation field $\sim 10^{4.5} G_{0}$, while the ratios of lower- $J \mathrm{CO}$ (i.e., $\mathrm{CO}(6-5)$ and $\mathrm{CO}(7-6))$ and ratios of higher- $J$ CO (i.e., $\mathrm{CO}(9-8)$ and $\mathrm{CO}(10-9)$ ) are overlapping with $L_{[\mathrm{O} \mathrm{I}]} / L_{[\mathrm{C} \text { II] }}$ and $L_{[\mathrm{C} \mathrm{II}]} / L_{[\mathrm{C} \mathrm{I}]}$ separately in regions with lower $G\left(\sim 10^{3} G_{0}\right)$. We also try to split the $\mathrm{CO}(6-5)$ and $\mathrm{CO}(7-6)$ lines based on the best-fit two-component model of COLSED in Figure 4, as shown in Figure 5 (Bottom panel). The "warm" components of $\mathrm{CO}(6-5)$ and $\mathrm{CO}(7-6)$ overlap with $\mathrm{CO}(9-8)$ and $\mathrm{CO}(10-9)$ at the region with high radiation field $\left(\sim 10^{5} G_{0}\right)$ and high density $\left(\sim 10^{5.5} \mathrm{~cm}^{-3}\right)$, while the "cold" components are overlapping with the ratios of $L_{[\mathrm{O} \mathrm{I}]} / L_{[\mathrm{C} \mathrm{II}]}$ and $L_{[\mathrm{C} \mathrm{II}]} / L_{[\mathrm{C} \mathrm{I}]}$ at the region having lower radiation field $\left(\sim 10^{3} G_{0}\right)$ and lower density $\left(\sim 10^{4.3} \mathrm{~cm}^{-3}\right)$. This result could be explained by a two-component model of CO lines, with a "cold" component dominated by the star formation and a "warm" component from other heating sources (e.g., AGN). However, we note that the simplified, one-cloud models of the ISM adopted here can be insufficient to encapsulate the full complexity of the ISM. In addition, the magnifications of different emission lines can introduce further complications.

\subsection{Water Vapor Emission}

Water vapor emission lines have been detected from $z>3$ galaxies and quasars, (e.g., van der Werf et al. 2011; Combes et al. 2012; Omont et al. 2013; Riechers et al. 2013). However, for $z>6$ quasars, only a tentative detection of two blended water emissions has been obtained (Bañados et al. 2015). From $\mathrm{J} 0439+1634$, two water vapor emission lines, $\mathrm{H}_{2} \mathrm{O} 33_{1,2}-2,1$ and $\mathrm{H}_{2} \mathrm{O} 3_{2,1}-3_{1,2}$, are detected from the NOEMA observations with high $\mathrm{S} / \mathrm{N}$ (see Figure 2). This is the first high-quality detection of multiple water vapor emission lines from quasar host galaxy at $z=6.5$.

A tight correlation between submm $\mathrm{H}_{2} \mathrm{O}$ lines $\left(L_{\mathrm{H}_{2} \mathrm{O}}\right)$ and total infrared luminosity $\left(L_{\mathrm{TIR}}\right)$ has been found in local and high-redshift infrared galaxies (Yang et al. 2013, 2016), although the relation between submm $\mathrm{H}_{2} \mathrm{O}$ emission and 

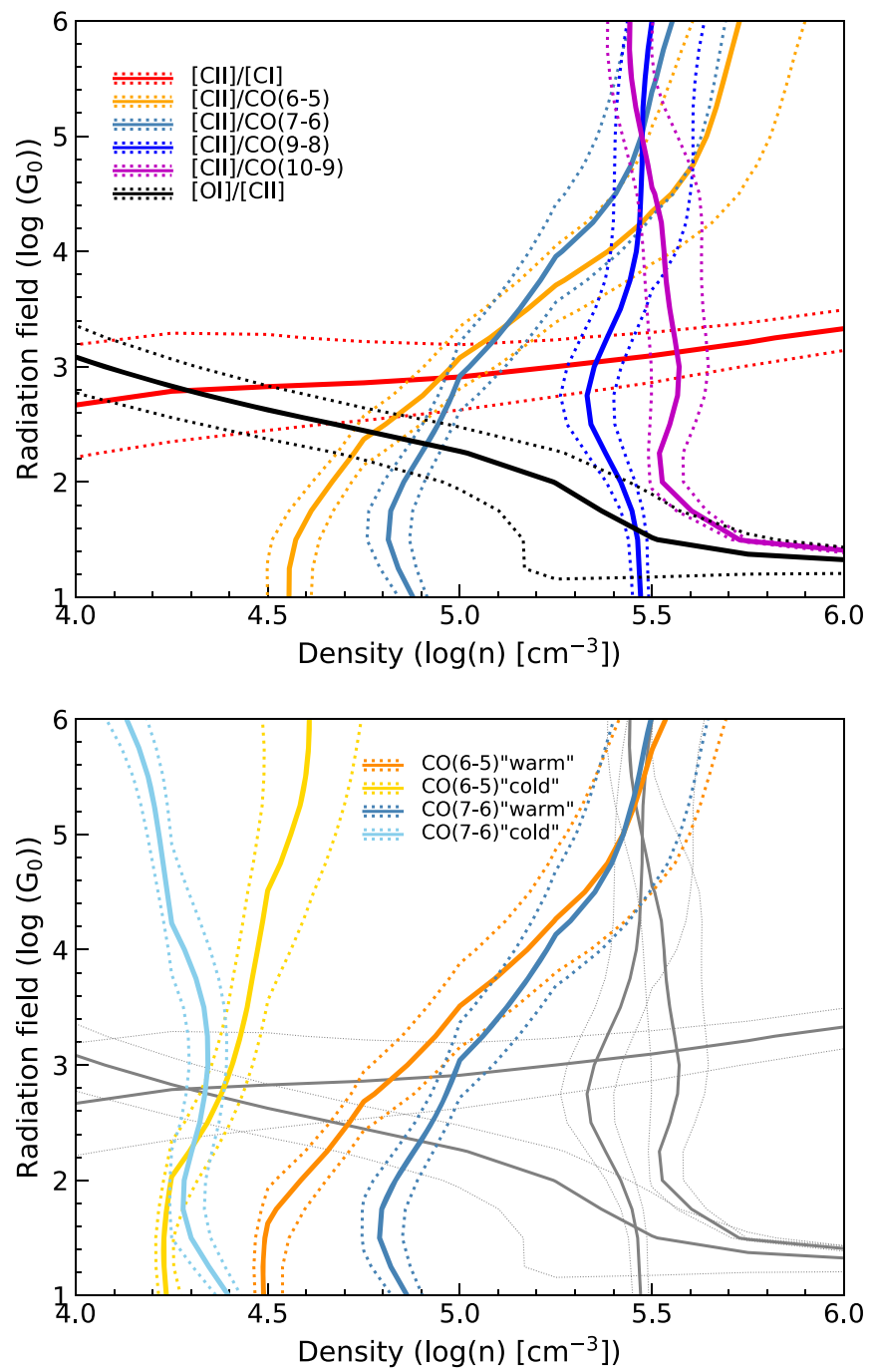

Figure 5. Top: line luminosity ratios of $L_{[\mathrm{C} \mathrm{m}]} / L_{\mathrm{CO}(6-5)}\left(=8.8\right.$, yellow), $L_{[\mathrm{C} \mathrm{m}} /$ $L_{\mathrm{CO}(7-6)}\left(=7.4\right.$, steel blue), $L_{[\mathrm{C} \mathrm{m}]} / L_{\mathrm{CO}(9-8)}(=4.1$, blue $), L_{[\mathrm{C} \mathrm{I}]} / L_{\mathrm{CO}(10-9)}$ (=4.2, purple), $L_{[\mathrm{C} \mathrm{I}]} / L_{[\mathrm{C} \text { I] }}\left(=24.3\right.$, red), and $L_{[\mathrm{O} \text { I] }} / L_{[\mathrm{C} \mathrm{I}]}(=0.3$, black $)$ of $\mathrm{J} 0439+1634$, compared with the predictions from the PDR model of Kaufman et al. (1999) in different conditions of gas density $n$ and radiation field $G$. Luminosities are all in units of $L_{\odot}$. The dotted lines are the $1 \sigma$ errors of line ratios. Bottom: the $L_{[\mathrm{C} \mathrm{II}} / L_{\mathrm{CO}}$ of split $\mathrm{CO}(6-5)$ and $\mathrm{CO}(7-6)$ lines compared with the other four ratios (gray) shown in the top panel. We split both the $\mathrm{CO}(6-5)$ and $\mathrm{CO}(7-6)$ lines into the "warm" and "cold" components based on the best-fit two-component model of COLSED in Figure 4 . The $L_{[\mathrm{C} \text { I] }} / L_{\mathrm{CO}}$ of "warm" $\mathrm{CO}(6-5)$ (dark orange) and $\mathrm{CO}(7-6)$ (dark blue) are consistent with the radios of $\mathrm{CO}(9-8)$ and $\mathrm{CO}(10-9)$ at the region with higher $G$ and $n$, while the radios of the "cold" $\mathrm{CO}(6-5)$ (yellow) and $\mathrm{CO}(7-6)$ (light blue) overlap with the $L_{[\mathrm{C} \mathrm{I}]} / L_{[\mathrm{C} \text { I] }}$ and $L_{[\mathrm{O} \mathrm{I}]} / L_{[\mathrm{C} \mathrm{I}]}$ at a region with a lower $G$ and $n$.

AGN activity is still unclear. Yang et al. (2016) suggested a correlation between $\mathrm{H}_{2} \mathrm{O} \quad 3_{2,1}-3_{1,2}$ line luminosity and total infrared luminosity as $L_{\mathrm{H}_{2} \mathrm{O}} /\left(10^{7} L_{\odot}\right)=\left(L_{\mathrm{TIR}} /\left(10^{12} L_{\odot}\right)\right)^{1.06 \pm 0.22}$, using a sample of local ultra-luminous infrared galaxies (ULIRGS) and high-redshift $(z \sim 2-4)$ ULIRGS or hyperluminous infrared galaxies. The luminosity of $\mathrm{H}_{2} \mathrm{O} 3_{2,1}-3_{1,2}$ detected of $\mathrm{J} 0439+1634$ is $7.4 \pm 1.4 \times 10^{8} \mu^{-1} L_{\odot}$. The ratio of $L_{\mathrm{H}_{2} \mathrm{O}} / L_{\mathrm{TIR}}$ follows the observed relation within scatter, as shown in Figure 6.

Previous studies (van der Werf et al. 2011; Yang et al. 2013, 2016) also show that strong AGN-dominated sources may have smaller average ratios than those in star-formingdominated galaxies. This could be explained by the very warm

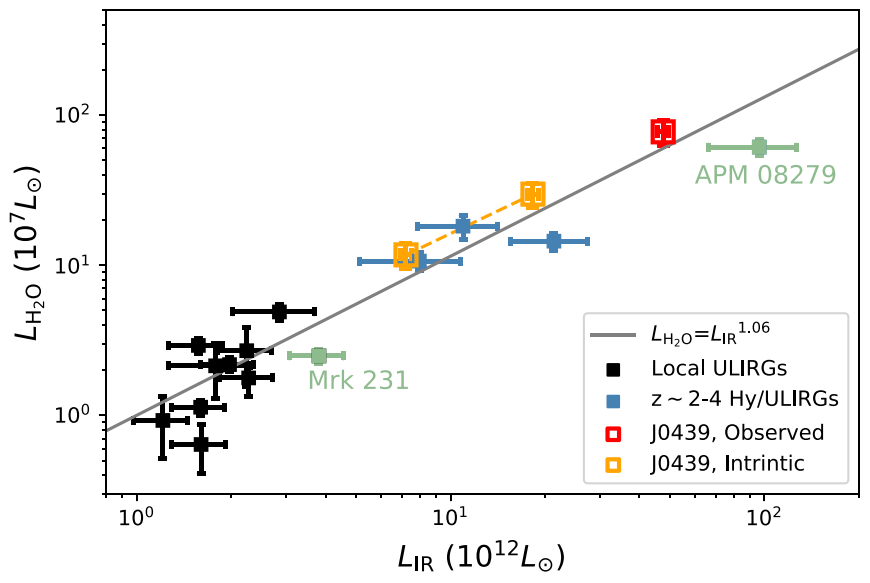

Figure 6. Luminosities of $\mathrm{H}_{2} \mathrm{O} \quad 3_{2,1}-3_{1,2}$ line and IR compared with the correlation (gray line) between $L_{\mathrm{IR}}$ and $L_{\mathrm{H}_{2} \mathrm{O} 321-312}$ of local ULIRGs (black squares) and high-redshift Hy/ULIRGs (blue squares) form Yang et al. (2013, 2016). The two green squares are sources excluded from correlation fitting in Yang et al. (2016) due to heavy AGN contamination. We plot them here for comparison. The red open square represent the $L_{\mathrm{IR}}$ and $L_{\mathrm{H}_{2} \mathrm{O} 321-312}$ before the magnification correction (observed), while the orange squares and dashed line denote the intrinsic $L_{\mathrm{IR}}$ and $L_{\mathrm{H}_{2} \mathrm{O} 321-312}$ assuming the $\mu_{\text {host }}$ is 2.6-6.6. As shown, J0439+1634 is following this correlation.

dust heated by the AGN. The higher dust temperature yields a higher IR luminosity, with only a modest increase in the rate of $\geqslant 75 \mu \mathrm{m}$ photons which dominates the excitation of $J \leqslant 3 \mathrm{H}_{2} \mathrm{O}$ emission lines (Kirkpatrick et al. 2015; Yang et al. 2016). The $L_{\mathrm{H}_{2} \mathrm{O}\left(3_{2} 1-31_{1}\right)} / L_{\mathrm{TIR}}$ ratio of $\mathrm{J} 0439+1634$ is $15.4 \times 10^{-6}$, slightly above the value based on the correlation of $\mathrm{Hy} /$ ULIRGS as shown in Figure 6 and higher than the mean values of both local AGN-dominated sources $\left(6.7 \times 10^{-6}\right)$ and local star-forming-dominated galaxies $\left(10.8 \times 10^{-6}\right)$ from Yang et al. (2016), which could indicate a significant contribution from star formation to the IR radiation of J0439+1634.

\section{Summary}

We report FIR and radio observations of the gravitationally lensed quasar J0439+1634 at $z=6.5$. Observations from NOEMA and JCMT have detected its dust continuum emission, multiple $\mathrm{CO},[\mathrm{CI}],[\mathrm{CI}]$, [O I], and water vapor emission lines with very high quality. Their properties make the host of J0439+1634 the brightest quasar host known at high redshift in the FIR. The VLA $3 \mathrm{GHz}$ imaging detects the radio continuum of $\mathrm{J} 0439+1634$ at rest frame $22.6 \mathrm{GHz}$. All these observations are spatially unresolved. The main results based on these observations are summarized as follows:

1. Based on multifrequency continuum emission detected from NOEMA and JCMT, we model J0439+1634's dust emission and estimate its FIR luminosity of $L_{\text {FIR }}$ $=(3.4 \pm 0.2) \times 10^{13} \mu^{-1} L_{\odot}$ with $\mu=2.6-6.6$, TIR luminosity of $L_{\mathrm{TIR}}=(4.8 \pm 0.2) \times 10^{13} \mu^{-1} L_{\odot}, \mathrm{SFR}_{\mathrm{TIR}}=7080$ $\mu^{-1} M_{\odot} \mathrm{yr}^{-1}$, and dust mass of $M_{\mathrm{d}}=(2.2 \pm 0.1) \times 10^{9} \mu^{-1}$ $M_{\odot}$.

2. The [C II] line detected of $\mathrm{J} 0439+1634$ is the brightest [C II] line detected from $z>6$ source, with a peak flux density of $\sim 36 \mathrm{mJy}$ and line luminosity of $L_{\text {[C II] }}=(1.2 \pm 0.1) \times 10^{10} \mu^{-1} L_{\odot}$. The [C II] line also provides en estimates of $L_{[\mathrm{C} \mathrm{II}]}$-based SFR, SFR ${ }_{[\mathrm{C} \mathrm{II}]}=$ $1000-6000 \mu^{-1.18} M_{\odot} \mathrm{yr}^{-1}$ and the mass of singly ionized carbon, $M_{\mathrm{C}^{+}}=(3.7 \pm 0.3) \times 10^{7} \mu^{-1} M_{\odot}$. The [C II] line 
also allows us to constrain its dynamical mass to first order.

3. With the four $\mathrm{CO}$ lines, $\mathrm{CO}(6-5), \mathrm{CO}(7-6), \mathrm{CO}(9-8)$, and CO(10-9), we model the COSLED of J0439+1634 with a LVG model and find that a two-component excitation model is needed to explain the observations of its molecular gas, although the constrain still has large uncertainty due to the lack of low- $J$ and very high- $J$ emission lines. Using the two-component model, we estimate the $\mathrm{CO}(1-0)$ line luminosity and further constrain the molecular gas mass to be $M_{\mathrm{H}_{2}, \mathrm{CO}}=5.4 \times 10^{10} \mu^{-1} M_{\odot}$.

4. The [C I] line in $\mathrm{J} 0439+1634$ allows us to estimate the atomic carbon mass of $M_{\mathrm{C} \text { I }}=(2.6 \pm 0.5) \times 10^{7} \mu^{-1} M_{\odot}$ and offers an independent constraint on the molecular gas mass, $M_{\mathrm{H}_{2}, \mathrm{C} \text { I }}=(3.9-8.9) \times 10^{10} \mu^{-1} M_{\odot}$. We find that the molecular gas masses of $\mathrm{J} 0439+1634$ derived from $\mathrm{CO}(1-0)$ luminosity and atomic carbon mass are consistent.

5. The line luminosity ratios between [C II], [C I], $\mathrm{CO}$, and [O I] places a first constraint on the radiation field and gas density of ISM in the host galaxy of J0439+1634. Comparing with the the predicted luminosity ratios from the PDR model of Kaufman et al. (1999), to explain the result of J0439+1634, a PDR model including more than one component is required.

6. The $L_{\mathrm{H}_{2} \mathrm{O}\left(3_{2,1}-3_{1,2}\right)} / L_{\mathrm{TIR}}$ luminosity ratio of $\mathrm{J} 0439+1634$ follows the $L_{\mathrm{H}_{2} \mathrm{O}\left(3_{2,1}-3_{1,2}\right)}-L_{\mathrm{TIR}}$ correlation of ULIRGS and is higher than the mean value of AGN-dominated sources, probably suggesting a signification contribution from star formation to FIR luminosity.

7. The VLA $3 \mathrm{GHz}$ observations indicate that $\mathrm{J} 0439+1634$ is a radio-quiet quasar with radio loudness $R=0.05-0.17$.

Future high resolution ALMA observations of J0439+1634 will fully resolve the [C II] and dust continuum emission of the quasar host galaxy and will refine the lensing model, allowing accurate measurements of the host dynamical mass and star formation surface density. Further high resolution imaging of multiple- $J$ CO emissions could spatially resolve the excitation condition of the molecular gas. In addition, future observations of multiple fine structure lines (e.g., [O I], [O III], [N II]) and molecular emissions (e.g., low- and high-J CO, HCN) will provide detailed measurements of ISM properties such as excitations and metallicities in the host galaxy of J0439+1634.

We thank Jianwei Lyu, George Rieke, Chentao Yang, and Chris Carilli for important discussions. J. Yang, X. Fan and M. Yue acknowledge support from the US NSF grant AST 1515115 and NASA ADAP grant NNX17AF28G. B.P.V. and F.W. acknowledge funding through the ERC grant "Cosmic Gas." C. Keeton acknowledges support from US NSF grant AST-1716585. X.-B. Wu thanks the support by the National Key R\&D Program of China (2016YFA0400703) and the National Science Foundation of China (11533001, 11721303). We are grateful to the JCMT and VLA for providing DDT observations. This work is based on observations carried our under project number S18DO and W18EI with the IRAM NOEMA Interferometer. IRAM is supported by INSU/CNRS (France), MPG (Germany), and IGN (Spain). The National Radio Astronomy Observatory is a facility of the National Science Foundation operated under cooperative agreement by Associated Universities, Inc.

Facilities: IRAM:NOEMA, JCMT, VLA.

\section{ORCID iDs}

Jinyi Yang (1) https://orcid.org/0000-0001-5287-4242

Bram Venemans (i) https://orcid.org/0000-0001-9024-8322

Feige Wang (i) https://orcid.org/0000-0002-7633-431X

Xiaohui Fan (1) https://orcid.org/0000-0003-3310-0131

Mladen Novak (iD https://orcid.org/0000-0001-8695-825X

Roberto Decarli (ib https://orcid.org/0000-0002-2662-8803

Fabian Walter (iD https://orcid.org/0000-0003-4793-7880

Minghao Yue (iD https://orcid.org/0000-0002-5367-8021

Emmanuel Momjian (i) https://orcid.org/0000-00033168-5922

Charles R. Keeton (iD https://orcid.org/0000-0001-6812-2467

Ran Wang (iD https://orcid.org/0000-0003-4956-5742

Ann Zabludoff (iD https://orcid.org/0000-0001-6047-8469

Xue-Bing Wu (iD https://orcid.org/0000-0002-7350-6913

Fuyan Bian (iD https://orcid.org/0000-0002-1620-0897

\section{References}

Bañados, E., Decarli, R., Walter, F., et al. 2015, ApJL, 805, L8 Bañados, E., Venemans, B. P., Mazzucchelli, C., et al. 2018, Natur, 553, 473 Beelen, A., Cox, P., Benford, D. J., et al. 2006, ApJ, 642, 694 Bertoldi, F., Carilli, C. L., Cox, P., et al. 2003a, A\&A, 406, L55 Bertoldi, F., Cox, P., Neri, R., et al. 2003b, A\&A, 409, L47 Carilli, C. L., Lewis, G. F., Djorgovski, S. G., et al. 2003, Sci, 300, 773 Chapin, E. L., Berry, D. S., Gibb, A. G., et al. 2013, MNRAS, 430, 2545 Colbert, J. W., Malkan, M. A., Clegg, P. E., et al. 1999, ApJ, 511, 721 Combes, F., Rex, M., Rawle, T. D., et al. 2012, A\&A, 538, L4 da Cunha, E., Groves, B., Walter, F., et al. 2013, ApJ, 766, 13 Decarli, R., Walter, F., Venemans, B. P., et al. 2018, ApJ, 854, 97 De Looze, I., Cormier, D., Lebouteiller, V., et al. 2014, A\&A, 568, A62 De Rossi, M. E., Rieke, G. H., Shivaei, I., Bromm, V., \& Lyu, J. 2018, ApJ, 869,4

Díaz-Santos, T., Armus, L., Charmandaris, V., et al. 2017, ApJ, 846, 32 Fan, X., Wang, F., Yang, J., et al. 2019, ApJL, 870, L11 Gullberg, B., De Breuck, C., Vieira, J. D., et al. 2015, MNRAS, 449, 2883 Ho, L. C. 2007, ApJ, 669, 821

Holland, W. S., Bintley, D., Chapin, E. L., et al. 2013, MNRAS, 430, 2513 Kaufman, M. J., Wolfire, M. G., Hollenbach, D. J., \& Luhman, M. L. 1999, ApJ, 527, 795

Kaufman, M. J., Wolfire, M. G., \& Hollenbach, D. J. 2006, ApJ, 644, 283

Kirkpatrick, A., Pope, A., Sajina, A., et al. 2015, ApJ, 814, 9

Lyu, J., Rieke, G. H., \& Alberts, S. 2016, ApJ, 816, 85

Maiolino, R., Cox, P., Caselli, P., et al. 2005, A\&A, 440, L51

Mazzucchelli, C., Bañados, E., Venemans, B. P., et al. 2017, ApJ, 849, 91

McGreer, I. D., Hall, P. B., Fan, X., et al. 2010, AJ, 140, 370

Meijerink, R., Spaans, M., \& Israel, F. P. 2007, A\&A, 461, 793

More, A., Oguri, M., Kayo, I., et al. 2016, MNRAS, 456, 1595

Murphy, E. J., Condon, J. J., Schinnerer, E., et al. 2011, ApJ, 737, 67

Nussbaumer, H., \& Storey, P. J. 1981, A\&A, 96, 91

Omont, A., Yang, C., Cox, P., et al. 2013, A\&A, 551, A115

Peng, C. Y., Impey, C. D., Rix, H.-W., et al. 2006, ApJ, 649, 616

Pound, M. W., \& Wolfire, M. G. 2008, adass XVII, 394, 654

Richards, G. T., Haiman, Z., Pindor, B., et al. 2006, AJ, 131, 49

Richards, G. T., Strauss, M. A., Pindor, B., et al. 2004, AJ, 127, 1305

Riechers, D. A., Bradford, C. M., Clements, D. L., et al. 2013, Natur, 496, 329 Rivera, J., Baker, A. J., Gallardo, P. A., et al. 2019, ApJ, 879, 95

Stacey, G. J., Geis, N., Genzel, R., et al. 1991, ApJ, 373, 423

Treu, T. 2010, ARA\&A, 48, 87

van der Tak, F. F. S., Black, J. H., Schöier, F. L., Jansen, D. J., \& van Dishoeck, E. F. 2007, A\&A, 468, 627

van der Werf, P. P., Berciano Alba, A., Spaans, M., et al. 2011, ApJL, 741, L38

Venemans, B. P., Bañados, E., Decarli, R., et al. 2015, ApJL, 801, L11

Venemans, B. P., Decarli, R., Walter, F., et al. 2018, ApJ, 866, 159

Venemans, B. P., Findlay, J. R., Sutherland, W. J., et al. 2013, ApJ, 779, 24

Venemans, B. P., McMahon, R. G., Walter, F., et al. 2012, ApJL, 751, L25

Venemans, B. P., Walter, F., Decarli, R., et al. 2017a, ApJ, 837, 146

Venemans, B. P., Walter, F., Decarli, R., et al. 2017b, ApJL, 851, L8

Venemans, B. P., Walter, F., Decarli, R., et al. 2017c, ApJ, 845, 154

Venemans, B. P., Walter, F., Zschaechner, L., et al. 2016, ApJ, 816, 37

Walter, F., Bertoldi, F., Carilli, C., et al. 2003, Natur, 424, 406

Walter, F., Carilli, C., Bertoldi, F., et al. 2004, ApJL, 615, L17 
Walter, F., Riechers, D., Cox, P., et al. 2009, Natur, 457, 699

Walter, F., Riechers, D., Novak, M., et al. 2018, ApJL, 869, L22

Walter, F., Weiß, A., Downes, D., Decarli, R., \& Henkel, C. 2011, ApJ, 730,18

Wang, R., Carilli, C. L., Beelen, A., et al. 2007, AJ, 134, 617

Wang, R., Wagg, J., Carilli, C. L., et al. 2011, AJ, 142, 101

Wang, R., Wagg, J., Carilli, C. L., et al. 2013, ApJ, 773, 44

Wang, R., Wu, X.-B., Neri, R., et al. 2016, ApJ, 830, 53

Wang, F., Yang, J., Fan, X., et al. 2018, arXiv:1810.11926
Weiß, A., Downes, D., Henkel, C., \& Walter, F. 2005, A\&A, 429, L25

Weiß, A., Downes, D., Neri, R., et al. 2007, A\&A, 467, 955

Weiß, A., Henkel, C., Downes, D., \& Walter, F. 2003, A\&A, 409, L41

Willott, C. J., Bergeron, J., \& Omont, A. 2015, ApJ, 801, 123

Yang, C., Gao, Y., Omont, A., et al. 2013, ApJL, 771, L24

Yang, C., Gavazzi, R., Beelen, A., et al. 2019, A\&A, 624, A138

Yang, C., Omont, A., Beelen, A., et al. 2016, A\&A, 595, A80

Yang, J., Wang, F., Fan, X., et al. 2019, AJ, 157, 236

Yun, M. S., Reddy, N. A., \& Condon, J. J. 2001, ApJ, 554, 803 